\title{
Uso de robots cooperativos para el desarrollo de habilidades de trabajo cooperativo en niños.
}

Resumen

\author{
Enrique González Guerrero ${ }^{1}$ \\ John Jairo Páez Rodríguez ${ }^{1}$ \\ Fabián José Roldán ${ }^{1}$
}

El presente trabajo describe una contribución al proyecto QUEMES. Es una propuesta para la educación en tecnología que aborda tres componentes: la construcción de los robots móviles diferenciales, el entorno gráfico de programación orientado a evento y las actividades pedagógicas para los niños. Este proyecto fue desarrollado en el 2010 por el grupo de investigación SIDRE de la Pontificia Universidad Javeriana en convenio con Maloka. QUEMES es una propuesta de aprendizaje en educación en tecnología usando robots cooperativos. El objetivo es que los niños aprendan a trabajar de forma cooperativa a través de la observación de cómo interactúan los robots para resolver un problema débilmente estructurado en forma conjunta. Se aplica la metodología de "Motivación para la creación", desarrollada por Maloka en sus clubes de ciencia; los problemas por solucionar parten de situaciones de la vida cotidiana, lo que facilita la asociación de la tecnología y su diario vivir. El software para la programación de los robots está desarrollado en un entorno gráfico que es muy fácil e intuitivo de utilizar. Los estudiantes sólo definen las interacciones entre los robots y las reacciones que estos deben tener ante los eventos; al compilar el programa se generan de forma automática los códigos necesarios para el control autónomo de los robots.

QUEMES, además de proporcionar una plataforma hardware y software, también plantea una serie de actividades tecnológicas escolares, donde los estudiantes aprenden los principios básicos de mecánica, electrónica y programación, necesarios para la comprensión del funcionamiento de los robots, además de reflexionar sobre la importancia del trabajo cooperativo. El aporte presentado consiste en extender las actividades educativas que los estudiantes pueden realizar mediante el diseño de nuevas tareas de cooperación entre los robots móviles, aprovechando la incorporación de un sistema prensil de objetos geométricos simples en cada robot.

Palabras clave: plataforma QUEMES, robótica, sistema multiagente, solución de problemas, trabajo cooperativo. 


\title{
Using cooperative robots to develop cooperative working
}

\section{skills in children.}

\begin{abstract}
This paper describes a contribution to QUEMES project. This is a proposal for education in technology approaching three parts: mobile differential robots construction, event oriented graphic programming environment and educational activities for children. This project was developed by Pontificia Universidad Javeriana Sidre research group in 2010, in agreement with Maloka. QUEMES is a technology education learning proposal using cooperative robots. The main goal is that the children learn to work in a cooperative way through observation of robot interaction to resolve a problem. "Motivation for the creation" was the applied methodology developed by Maloka. The problems to resolve were every day-life situations facilitating the association between technology and daily life. The software uses for programming the robots was developed in a highly intuitive and easy-to-use graphic environment. The students define the interactions and reactions between robots to the events. When compiling the programs the code for the robot standalone control system was generated automatically.

QUEMES aside from providing a hardware and software platform, propose a number of technological school activities where the students learn mechanics electronics and basic principles of computer programing, required for understanding of how robots work besides of think over cooperative work. The main contribution presented is the extension of educational activities that the students can do through the design of new cooperation tasks within robots, using the simple geometric objects prehensile system.
\end{abstract}

Key words: QUEMES platform, robotics cooperative work, multiagent system, problem solving, QUEMES platform, robotics. cooperative work.

\section{Introducción}

El siguiente artículo presenta, inicialmente, los antecedentes relacionados con el uso de robots cooperativos en la educación. Las características físicas de los robots de la plataforma QUEMES en donde se mencionan los aspectos mecánicos y electrónicos más significativos. Después, se aborda el diseño del software de la plataforma, el cual se desarrolla dentro del paradigma de los 
sistemas multiagente y la programación orientada a eventos. Finalmente, se presentan las actividades educativas propuestas, las conclusiones relacionadas con su implementación y un espacio de discusión para trabajos futuros.

\section{Antecedentes}

De acuerdo con los trabajos previos, es evidente la ausencia de estrategias de educación en tecnología que utilizan robots cooperativos en la enseñanza del mismo trabajo cooperativo. El único antecedente encontrado y mencionado es el proyecto QUEMES (González, et al., 2010), donde de manera incipiente se pretende utilizar los robots como instrumento de reflexión para que los estudiantes al observar el trabajo en forma cooperativa de los robots, puedan interiorizar estos comportamientos en la vida cotidiana. En cuanto a sus ventajas en la solución de problemas con matices tecnológicos, no se plantea de forma clara como este tipo de trabajo permite desarrollar las competencias necesarias para trabajar en equipo y menos aún, como este tipo de trabajo puede mejorar las competencias ciudadanas de los estudiantes. Esta carencia puede ser causada porque hasta ahora se están desarrollando plataformas de educación en tecnología para niños, que utilizan los sistemas multiagentes como estrategia didáctica.

Actualmente en la educación, los robots móviles se utilizan para la comprensión de distintas áreas del conocimiento. Ejemplos de este tipo de desarrollos son presentados en Slovakia (Petrovic y Balogh, 2008) y Taiwan (Liu, 2010), donde hasta hace pocos años, se está implementando diferentes cursos y congresos de robótica para incentivar a los estudiantes y las instituciones a trabajar con este tipo de tecnología. En el caso colombiano existe una tendencia popular de mencionar la palabra robótica como medio atractivo para la enseñanza de la tecnología, pero de acuerdo con el análisis de antecedentes, son pocas las instituciones (González, et al., 2010) que se dan a la tarea de formalizar estrategias pedagógicas. En la mayoría de los casos, las propuestas sólo se limitan a crear grupos de estudiantes en entornos educativos formales y noformales para que construyan algunas aproximaciones muy limitadas de robots que no hacen uso de la inteligencia artificial en el diseño del comportamiento de los robots.

Un ejemplo de este tipo de aproximaciones lo se ve en el trabajo denominado Advanced Robotics Projects for Undergraduate Students (Blank, 2007), en el cual la propuesta técnica de desarrollar entornos virtuales para trabajar con robots es clara, pero el tema pedagógico es nuevamente muy incipiente. De igual manera, la mayoría de proyectos consultados, como Robots Byte In: An Exploration of Computer Science Education in Middle Schools (Gupta, et al., 2010), centran la discusión del trabajo cooperativo en términos de colocar 
a varios estudiantes para el trabajo con el mismo robot, pero realmente es muy escasa la reflexión acerca de los componentes pedagógicos del trabajo cooperativo y además no se usan robots cooperativos en la propuesta. De la reflexión anterior, se concluye que las investigaciones para el aprendizaje de trabajo cooperativo con robots cooperativos son escasas y, por este motivo, este trabajo muestra cómo articular estos dos elementos.

\section{Características de los robots quemes.}

Los robots de la plataforma QUEMES son robots diferenciales, compuestos por dos servomotores, cuatro sensores infrarrojos, un microcontrolador, un módulo de comunicación inalámbrica, la estructura metálica y el sistema prensil. Este último es el aporte de este trabajo el cual obligó a hacer modificaciones en la arquitectura del programa JAVA, encargado, por un lado, de establecer parámetros de comunicación con el usuario y además, de ejecutar la plataforma BESA que permite el control de los robots de acuerdo con el paradigma de los sistemas multiagente (Foster, 1999; Ferber, 1999). También fue necesario hacer modificaciones en el sistema embebido que permite el control del hardware. A continuación se presenta el modelo estructural del robot sin sistema prensil y de forma ampliada, el diseño de la pinza.

Gráfica 1: Estructura mecánica del robot y la pinza.

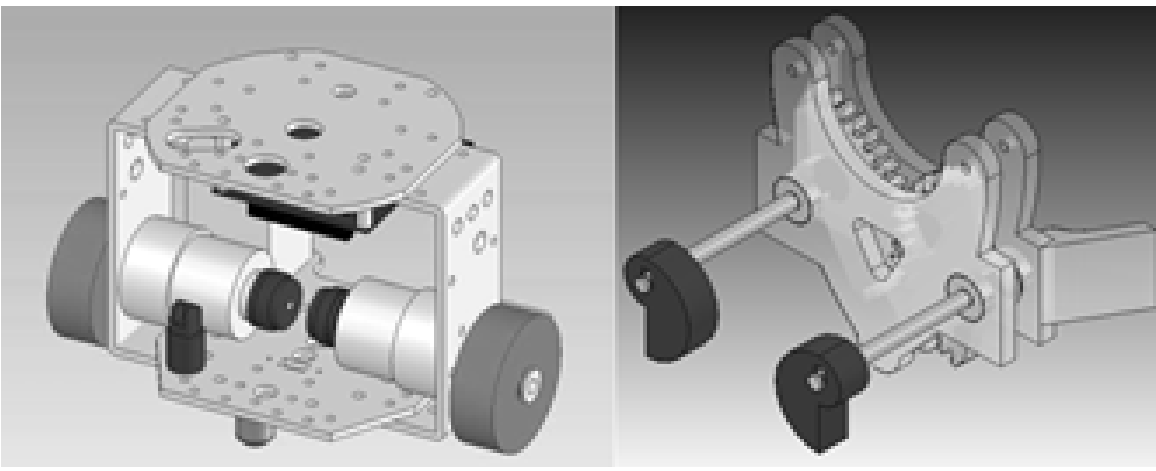

Fuente: Autores 
Teniendo en cuenta el análisis de los robots de la plataforma QUEMES, en donde ya hay un gran avance en términos de desarrollo técnico de los robots y de la plataforma para su programación en relación al trabajo cooperativo, se ha propuesto la ampliación de las actividades de cooperación por medio de la implementación en cada robot, de un sistema prensil dotado de sensores de color. Con este sistema los niños tienen más oportunidades para desarrollar actividades cooperativas en los robots, lo que incide de manera significativa en el desarrollo de las habilidades sociales.

La implementación del sistema prensil permite a los robots modificar el ambiente, ya que pueden tomar diferentes objetos geométricos para generar nuevos estados y condiciones que amplían las actividades de cooperación. El diseño mecánico de la pinza permite con un solo motor sujetar un objeto y al mismo tiempo levantarlo. Para el diseño mecánico se tuvo en cuenta la limitación en el consumo de energía y, por tal motivo, surge el desafío de utilizar un solo motor para generar dos acciones paralelas en la pinza; sujetar el objeto y desplazarlo hacia arriba. El sistema prensil cumple las siguientes funciones en la plataforma QUEMES:

Función comunicativa, la interacción entre los robots aumentará dada una mayor correspondencia entre una serie de mensajes que definen el estado de los robots en función de la manipulación de los objetos.

Función dinámica, el sistema prensil permite el despliegue en tiempo real, de un proceso individual y cooperativo en el que van cambiando diferentes parámetros, estos cambios pueden ser de orden perceptual, espacial y cinético. Perceptual, dado que las representaciones jerárquicas reconfiguran los procesos del control de movimiento en cada robot. Espacial, porque las actividades de programación para la coordinación en la toma de objetos geométricos simples, depende de las condiciones dinámicas del robot y su respectivo sistema de control, ya que debe tener funciones de orientación que le permita crear formas dinámicas en relación con el movimiento antes de entrar en contacto. Cinético, porque el entrenamiento de coordinación pinza y robot produce cambios en los planos de referencia continuos del robot y movimientos de los planos de referencia.

\section{Metodología de trabajo con QUEMES}

La plataforma QUEMES es una herramienta pedagógica que le permite a los usuarios ver reflejado el diseño y la programación orientada a eventos bajo el paradigma de computación de sistemas multiagentes de manera gráfica y muy intuitiva, inicialmente desarrollada por los grupos de investigación SIDRe y Takina de la Pontificia Universidad Javeriana, GIAP de la Universidad de los Andes y Maloka (González et al., 2010). Para la implementación de control de los robots se utilizó la plataforma BESA, que es una plataforma desarrollada en el 
Departamento de Ingeniería de Sistemas de la Pontificia Universidad Javeriana y que facilita la implementación y ejecución de sistemas multiagentes (SMA). El acrónimo: BESA toma su nombre en inglés y encapsula los conceptos: Behavior-Oriented, Event-Driven, Social-Based, Agent Framework; los cuales son los principios sobre los que se fundamenta las soluciones creadas a partir de la plataforma.

A continuación se da una idea básica de cada principio (González, et al., 2003; González, et al., 2006). La plataforma QUEMES permite a los usuarios crear SMAs por medio del establecimiento de las pautas de comunicación entre los robots (caracterización de vínculos) y conformación de la cooperación (asignación de responsabilidades por medio de los roles, y definición de comportamientos). QUEMES gira en torno a los conceptos generales de los sistemas multiagente como cooperación, coordinación, comunicación, solución de conflictos y colaboración, los cuales se reflejan en las diferentes herramientas del programa como: creación de agentes, definición de roles, comunicación de roles uno a uno, comunicación a sí mismo, uno a varios, etc.

Gráfica 2: Entorno de programación desarrollado.

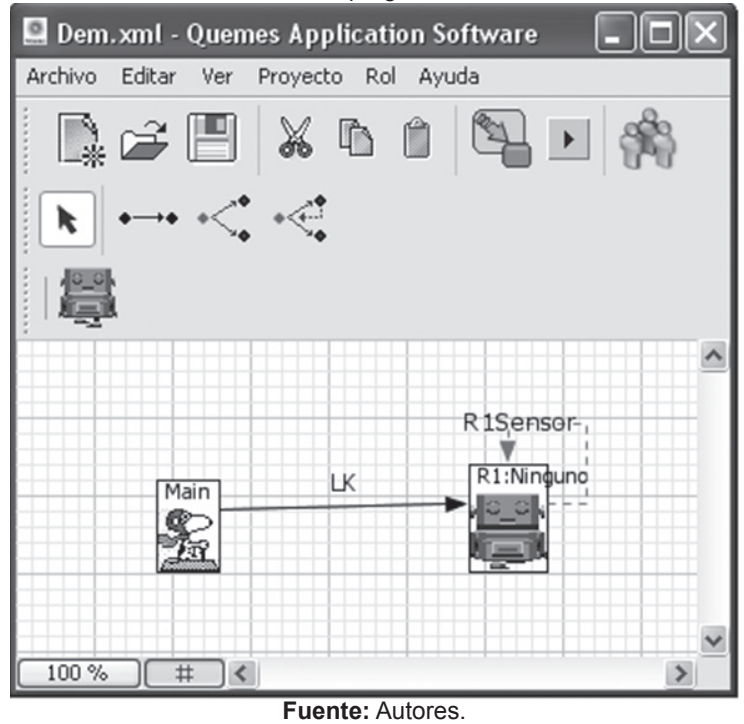

En QUEMES, para posibilitar la comunicación entre robots, es necesario definir las conexiones (o links) de las cuales existen cuatro tipos.

- Link Robot a Robot: define una comunicación unidireccional, donde un robot emisor envía unos datos al robot receptor.

- Link Rol a Rol: la comunicación rol a rol involucra una comunicación unidireccional, con la característica adicional de ser multicast por tratarse de una comunicación de varios a varios. 
- Link Rol a Rol Puntual o Respuesta: se presenta en el contexto de una conexión Rol a Rol, cuando un robot de un rol en particular requiere responder al emisor de la conexión Rol a Rol.

Cada conexión representa un evento del entorno de trabajo hacia el robot; por lo tanto, es necesario definir la reacción de todos los eventos involucrados. Para definir las reacciones de los eventos, en primera instancia se consideró la reacción como un conjunto de instrucciones ordenadas (un algoritmo), expresada en términos de un diagrama de flujo (con el fin de generalizar el uso de la herramienta, para aquellos usuarios que no cuentan con conocimientos específicos en $\mathrm{x}$ lenguaje de programación). QUEMES para proveer la funcionalidad de definición de reacciones (edición del diagrama de flujo), se comunica con un módulo de una aplicación desarrollada en Maloka, denominada Percival. Este último permite la edición de la estructuras de control del diagrama de flujo, a manera de drag and drop. Adicionalmente, provee, al usuario, de un conjunto de funciones predefinidas, las cuales están relacionas con el mundo físico de los robots. Funciones tales como avanzar y seguir línea, girar a la derecha y girar a la izquierda.

Gráfica 3: Percival, herramienta desarrollada para la programación de los robots por parte de los niños.

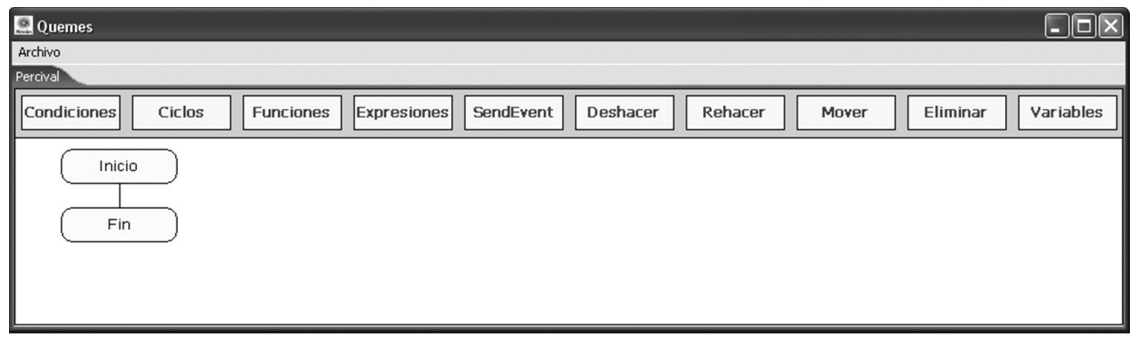

Fuente: Autores.

La arquitectura de QUEMES consta de tres capas: capa de presentación, en donde se establecen los requerimientos para interactuar con los usuarios; capa lógica, soportada por cuatro agentes: agente manejador de eventos, agente manejador de proyectos, agente manejador de robots y agente manejador de vínculos; y la capa de datos, que comparten dos capas anteriores.
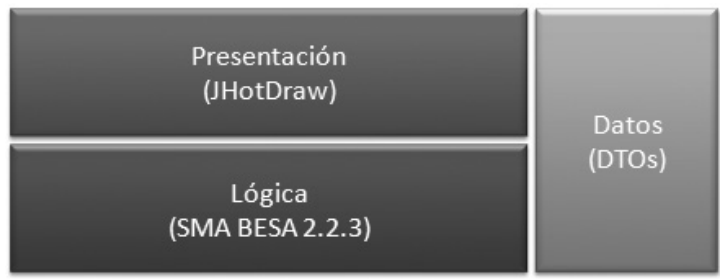

Gráfica 4: Arquitectura de QUEMES. 
Finalmente, se procede a generar, construir y a ejecutar el proyecto. En la ejecución, se despliega un diálogo de control, el cual permite al usuario controlar el comportamiento de los robots.

\section{Modelo pedagógico}

El desarrollo de las actividades con la plataforma QUEMES para la solución de diferentes problemas, utiliza la estrategia planteada por Maloka llamada «Motivación para la Creación» y los principios del aprendizaje cooperativo. Para el trabajo con los robots se han planteado cuatro momentos:

- Etapa de sensibilización.

- Etapa de fundamentación.

- Etapa de diseño y desarrollo de las actividades con los robots.

- Etapa de evaluación.

La etapa de sensibilización acerca a los niños en temas cotidianos que están presentes en la ciudad y donde se pueden tomar varios aspectos como pretexto para el desarrollo de actividades educativas con contenido técnico y social relacionado con el desarrollo de competencias ciudadanas. La etapa de fundamentación, retoma las actividades propuestas en la anterior etapa y le da un sentido académico donde se puede puntualizar en contenidos temáticos acorde con el área de tecnología, específicamente el tema de robots cooperativos y el desarrollo de competencias sociales.

La tercera etapa busca contextualizar la programación de los robots de forma cooperativa con las situaciones identificadas en el contexto de la ciudad y que atiende a los requerimientos del programa de Ciudad-Escuela-Ciudad del programa Bogotá positiva. Finalmente, en la etapa de evaluación, la cartilla propone una serie de actividades de reflexión donde los estudiantes pueden hacer transferencia del conocimiento aprendido en las etapas anteriores, en situaciones sociales que requieren el trabajo en equipo y la puesta en práctica de competencias ciudadanas.

A continuación se presentan las características de implementación para la simulación del problema propuesto a los niños de una ciudad en emergencia. Se tiene una ciudad divida en cuatro zonas y cada zona es controlada por un agente llamado agente zona. En el caso de una emergencia (robo, herido, incendio), el agente zona informa a un agente de la ciudad llamado central 123 sobre esa emergencia y las coordenadas donde ocurre. Ahora, el agente central 123 dependiendo del tipo de emergencia, le informa al agente central ( $\mathrm{p}, \mathrm{a}, \mathrm{b})$ policía, ambulancia o bombero, la zona donde ha ocurrido la emergencia y de acuerdo al método Contract Net se selecciona el agente más apropiado para atenderlo y si es el caso, tomarlo con la pinza. 
Para el movimiento de los agentes (policía, ambulancia o bombero), el agente zona maneja la información del ambiente e informan a los estos agentes el estado del mundo. Entonces, el agente que se desea mover le envía un evento al agente zona y le dice: «me quiero mover» y el agente zona le envía las condiciones de la zona y las posibilidades de movimiento. Ahora bien, el agente (policía, ambulancia o bombero) debe decidir el movimiento, el cual está en función de la distancia de donde es requerido por el evento (robo, herido, incendio). Durante el movimiento el agente va actualizando su posición de manera que el agente zona pueda saberla y evitar posibles conflictos con otros agentes.

El proceso de navegación de cada agente por la ciudad se desarrolla de la siguiente manera: El agente ( $\mathrm{p}, \mathrm{a}, \mathrm{b}$ ) ya tiene conocimiento que ha sido elegido para atender la emergencia, entonces el agente central ( $\mathrm{p}, \mathrm{a}, \mathrm{b})$ inicializa la variable sensor con las coordenadas de la emergencia, después se inicia el proceso de desplazamiento hacia la meta el cual está conformado por los siguientes eventos:

\section{While (emergencia sin atender )}

*El agente $(\mathrm{p}, \mathrm{a}, \mathrm{b})$ solicita al agente zona ver las condiciones del entorno, es decir, si hay o no obstáculos.

*El agente zona le envía la información del entorno relacionada con las cuatro celdas [N,E,S,O] vecinas a la ubicación del agente $(\mathrm{p}, \mathrm{b}, \mathrm{a})$.

*El agente (p,b,a) actualiza el conocimiento que tiene sobre su estado en el entorno y toma la decisión de movimiento.

*El agente zona actualiza el estado del agente $(p, b, a)$ del entorno.

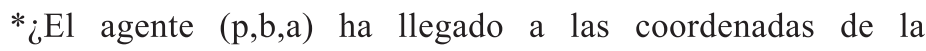
emergencia?

\section{End}

- Solución de la emergencia.

- Retornar a la posición indicada. 
Enrique González Guerrero, John Jairo Páez Rodríguez, Fabián José Roldán

Uso de robots cooperativos para el desarrollo de habilidades de trabajo cooperativo en niños. Artículo producto de la investigación.

Gráfica 5: Estado de movimiento de los agentes móviles.

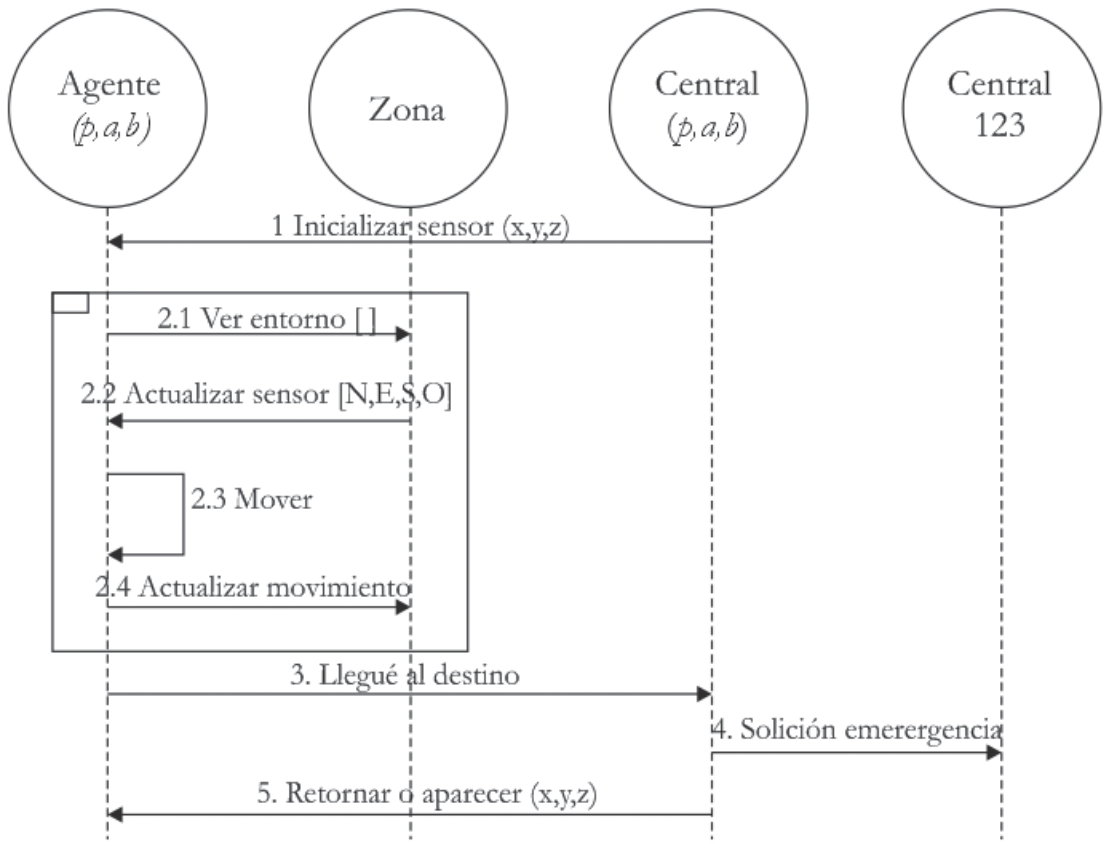

Gráfica 6: Modelo abstracto de la simulación de la ciudad en emergencia.
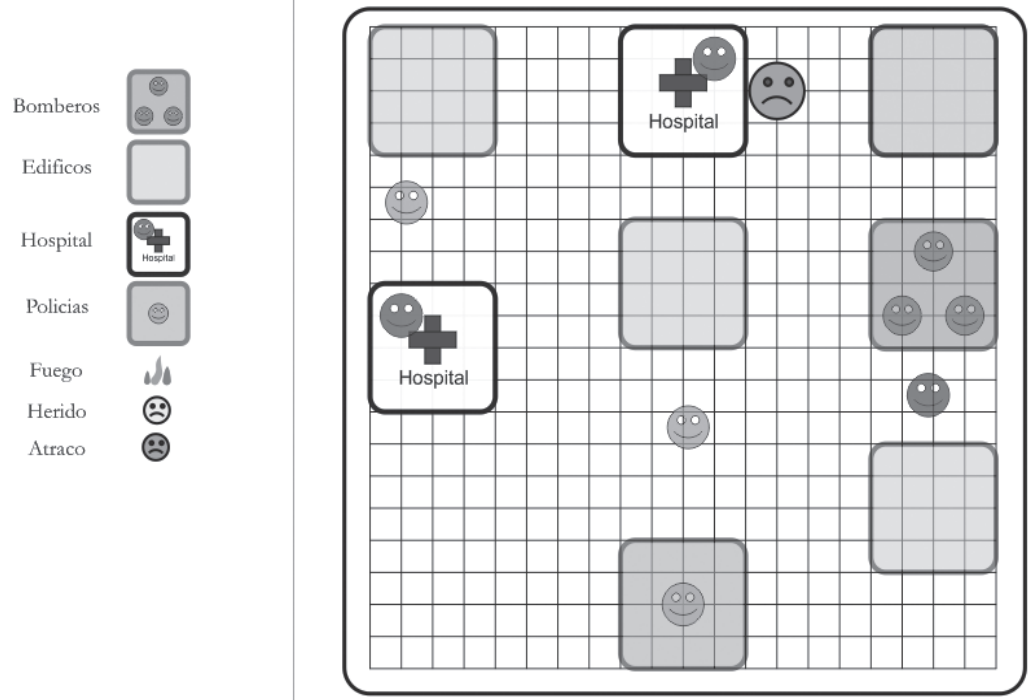


\section{Elementos de cooperación de QUEMES que motivan la reflexión en los estudiantes}

Dentro del desarrollo de la simulación de emergencia con sistemas multiagente, hay aspectos que validan la cooperación entre los agentes y que se utilizan en la reflexión pedagógica con los estudiantes. El primer elemento es la interacción, que se presenta en los meta agentes zona, central 123, central policía, central ambulancia y central bomberos. Dadas las características presentadas en la descripción del problema, se puede afirmar que los agentes: zona, central 123 , central $(p, a, b)$ y agentes $(p, a, b)$ tienen objetivos compatibles, recursos insuficientes y habilidades suficientes que permiten implementar métodos entre los agentes $(p, a, b)$ para la solución de conflictos generados por una eventual petición simultánea del espacio de movimiento y que se soluciona con un método de sincronización diseñado en BESA. Pero dado el caso, los eventos de robo, heridos e incendios se han diseñado con la misma prioridad de atención de tal manera que la regulación para el acceso a los recursos (las vías) es solucionada por la jerarquía de los agentes central 123, zona y central (p,a,b).

Otro elemento es la coordinación del agente 123 con los agentes zona y agentes central $(\mathrm{p}, \mathrm{a}, \mathrm{b})$. Durante el desarrollo de la tarea, los agentes, además de no presentar redundancia, constantemente se comunican con el agente zona para actualizar el conocimiento que tienen del mundo y así evitar que se presenten conflictos entre los agentes móviles. Un ejemplo es en el primer nivel en donde los agentes (p,a,b), navegan simultáneamente para atender las diferentes emergencias y en las cuales se favorece el manejo de acciones concurrentes, se evitan colisiones y demoras.

La comunicación propuesta de carácter intencional, es un proceso que se aborda en dos aspectos: broadcasting (hay un evento en una zona $\mathrm{n}$ y se necesita la presencia de una agente) y de enrutamiento directo (un agente central $(p, a, b)$ le indica a un agente $(p, a, b)$ atender una emergencia) que se ha mostrado de manera implica en el desarrollo del proyecto. Sin embargo, se puede encontrarla de manera muy precisa en el proceso de difusión que hacen los agentes central $(p, a, b)$ en la subasta para la selección del agente indicado en la atención de las emergencias. Un elemento importante de resaltar es que la comunicación que se maneja en el desarrollo del proyecto es únicamente de dos tipos: asertiva y directiva.

Dentro de los parámetros de diseño para la cooperación, se ha tenido en cuenta la implementación de agentes especializados con las habilidades y recursos necesarios en la atención de la emergencia. Este criterio, justifica el proceso de asignación centralizada y la presencia de una estructura jerárquica de subordinación. Aquí, los agentes conocen sus habilidades y no pueden atender otro tipo de emergencias para las cuales han sido diseñados. Además, el programa está diseñado para asegurar que los estados del contrato; asignación, desarrollo, concluido y ejecutado no se alteren en la asignación de nuevos eventos de emergencia. 


\section{Resultados y discusión}

La discusión de este trabajo se plantea desde dos perspectivas: primera, las características técnicas de los robots y, segunda, las ventajas pedagógicas. En relación con las características técnicas, se puede afirmar que la plataforma QUEMES es robusta en tanto el sistema embebido en el robot, controlado bajo el paradigma de RTOS y la plataforma desarrollada en JAVA, que cuenta con la arquitectura BESA elaborada por la Universidad Javeriana, son altamente compatibles y esto aumenta la tolerancia a fallos. Sin embargo, se plantea la posibilidad de optimizar la herramienta de programación Percival.

Desde el ámbito pedagógico, se puede afirmar que QUEMES presenta una gran cantidad de alternativas de trabajo de cooperación con los robots, más aun teniendo en cuenta que el sistema prensil implementado ha generado nuevas posibilidades de cooperación de los robots, lo que incide en las actividades de reflexión de los niños en torno a las bondades del trabajo cooperativo, la inteligencia colectiva, la cognición distribuida y demás características de la sociedad informacional soportada en el paradigma pedagógico del conexionismo y el trabajo en red.

El desarrollo de esta propuesta generó gran motivación en los estudiantes. A la fecha, después de un proceso de conceptualización, se cuenta con una aplicación que permite caracterizar robots, vínculos de comunicación y reacciones con el fin de facilitar la construcción de SMAs de una forma rápida y sencilla, sin la necesidad de que el usuario cuente con conocimientos avanzados en programación. Inicialmente, el proyecto QUEMES solo interactuaba con el entorno a través de los sensores, ahora con la implementación de la pinza, se han ampliado las posibilidades de cooperación que enriquece su propuesta pedagógica y didáctica.

El uso de robots cooperativos en la educación parte desde la perspectiva cooperativa y social, aunque es necesario aclarar que el tema no está muy avanzado en el mundo, en Colombia se toman en cuenta dos aspectos. El primero, los estándares básicos de competencias ciudadanas del Ministerio de Educación Nacional, donde se entienden como una herramienta para ejercer los derechos y saberes de un buen ciudadano y que además, tiene cuatro objetivos fundamentales: aprender a vivir juntos, trabajar en equipo, identificar particularidades y diferencias. El segundo, desarrollar procesos de interacción social. En este sentido, las competencias ciudadanas se conciben como un conjunto de conocimientos, habilidades cognitivas, emocionales y comunicativas que permiten resolver cualquier situación social cotidiana. Para ampliar estos términos se puede afirmar que los conocimientos representan la información que los niños deben tener acerca del ejercicio de la ciudadanía, 
las habilidades cognitivas hacen referencia a la capacidad de reflexión y anticipación que deben tener los estudiantes para proveer las consecuencias de sus actos en entornos sociales, las competencias emocionales son las habilidades necesarias para el control de las emociones personales e interpersonales y, las competencias comunicativas que hacen referencia a la habilidad de expresarse con claridad, sin agresión a los demás y con capacidad para escuchar a los otros.

En el contexto del desarrollo de este proyecto, ya se han realizado en varios colegios de la ciudad de Bogotá, diferentes actividades que dan validez a la investigación, en donde se evidencia una gran acogida por parte de los estudiantes dado que la mayoría demuestran un gran interés y motivación durante el proceso de aprendizaje de los conceptos de tecnología y el desarrollo de habilidades sociales y competencias ciudadanas. De acuerdo con lo anterior se puede afirmar:

Las personas que desarrollen la propuesta pueden tener una mirada más holística cuando se enfrenten a un problema relacionado con la tecnología o con las competencias ciudadanas. El desarrollo de las habilidades sociales aporta de manera significativa al desarrollo de un país. Estas características sociales, que los niños hacen evidente en la programación de los robots, se pueden utilizar para reflexionar sobre el comportamiento responsable frente a los demás, la responsabilidad ética del trabajo y la responsabilidad personal, donde los medios y los fines, presentan una dimensión teórica relacionada con las normas y los principios, y una dimensión practica relacionada con los hábitos y las virtudes. En este sentido, se espera que la relación entre trabajo cooperativo y comportamiento de cooperación de los niños durante la programación de los robots, permita generar espacios de reflexión que produzcan mejoras significativas en sus comportamientos éticos. 


\section{Referencias bibliográficas}

Blank, D., Kummar, D., Marshall, J. y Meeden, L. 2007. Advanced Robotics Projects for Undergraduate Students. Computer Science Program Bryn Mawr College.

Denis, B. y Hubert, S. 2001. Collaborative Learning in an Educational Robotics Environment. Computers in Human Behavior .17: 465-480.

Ferber, J. 1999. MultiAgent Systems: an Introduction to Distributed Artificial Intelligence. New York: Addison Wesley.

Foster, I. 1995. Designing and Building Parallel Programs. New York. Addison Wesley, 1995.

González, E., Bustacara, C., Roldán, F. Buitrago, S., Avellaneda, M. y Fajardo, L. 2010. QUEMES: Technology Education based on Cooperative Robots. Bogotá: Pontificia Universidad Javeriana / Maloka.

González, E., Pérez, A., Torres, M., Garzón, J., Rodríguez, J., Cruz, J. D., Mancilla, L., Cantor, O., Ahogado, D., y Reinemer, A. 2006. Desarrollo de aplicaciones basadas en sistemas multiagentes. Bogotá: Pontificia Universidad Javeriana.

González, E., Ávila, J. y Bustacara, C. 2003. BESA: Behavior-oriented, Event-driven and Social-based Agent Framework. Bogotá: Pontificia Universidad Javeriana.

González E., Ávila J., Bustacara C. 2003. BESA: Arquitectura para la construcción de sistemas multiagentes. 2003. Bogotá: Pontificia Universidad Javeriana.

Liu, E., Lin, Ch., Chang, Ch. 2010. Student Satisfaction and Self-Efficacy in a Cooperative Robotics Course. Social Behavior and Personality and International Journal. 39 (8): 1135-1146.

Mitnik R., Recabarren, M., Nussbaum, M, Soto, A. 2009. Collaborative Robotic Instruction: a Graph Teaching Experience. Computers \& Education. 53 (2): 330-342.

Petrovic, P., Balogh,R. 2008. Educational Robotics Initiatives in Slovakia, Workshop Proceedings of SIMPAR. Intl. Conf. on Simulation, Modeling And Programming For Autonomous Robots, Venice(Italy) 2008 November,3-4. 